\title{
Welcome to the Education in Medicine Journal
}

The Education in Medicine Journal (EIMJ) published its first publication a year ago, on December 2009. It is a very new online journal in the field which we hope will attract medical and allied health colleagues locally and internationally who have found it difficult to publish papers in the major medical education journals and who may have English as a second language. We hope that the EIMJ can be a solution to this situation and provide a useful forum where medical and allied health educators, both basic scientists and clinicians, from around the world can share educational ideas and developments.

The focus of the journal will be the areas of medical and allied health education as listed in the 'about us' section. We are particularly interested in papers that have explored the impact of student development programmes on medical and allied health students' personal and professional development. We wish to promote the exchange of ideas and experiences that derive from the different cultures in which the student development programmes occur throughout the world. This may contribute towards the internationalisation of student development principles.
Apart from our specific interest on the impact of student development programmes on students from multicultural and multi-ethnic backgrounds, we also welcome papers from various areas of medical and allied health education such as teaching and learning, educational environment, assessment, student selection, psychometric development related to medical and allied health education and other areas which may be of interest.

In order to further this universal philosophy the journal will publish all articles free of charge which we hope will encourage more authors to share their work with the medical and allied health education community. On top of that we are currently working toward indexing the journal in reputable databases in future.

Muhamad Saiful Bahri Yusoff, MD, MScMEd

Medical Education Department, School of Medical Sciences, Universiti Sains Malaysia

Kubang Kerian, Kelantan, Malaysia Editor

Email: $\underline{\text { msaiful@kb.usm.my }}$

Ahmad Fuad Abdul Rahim, MD, MPHEd

Head, Medical Education Department, School of Medical Sciences, Universiti Sains Malaysia, Kubang Kerian, Kelantan, Malaysia

Associate Editor

Email: fuad@kb.usm.my 